\title{
ACUTE KIDNEY INJURY AND CHRONIC KIDNEY DISEASE IN HIV PATIENTS IN THE ERA OF ANTI- RETROVIRAL THERAPY
}

\author{
Priya V. Patil1, Jyoti B. Bansode2, Manoj C. Kore ${ }^{3}$, Kashmira Hajare4
}

${ }_{1}^{1}$ Associate Professor, Department of General Medicine, Grant Medical College and Sir JJ Hospitals, Mumbai, Maharashtra.

2Senior Resident, Department of General Medicine, Grant Medical College and Sir JJ Hospitals, Mumbai, Maharashtra.

${ }^{3}$ Senior Resident, Department of General Medicine, Grant Medical College and Sir JJ Hospitals, Mumbai, Maharashtra.

${ }_{4}^{4}$ Assistant Professor, Department of General Medicine, Grant Medical College and Sir JJ Hospitals, Mumbai, Maharashtra.

\section{ABSTRACT}

\section{BACKGROUND}

HIV/AIDS may have renal involvement as a direct consequence of HIV infection, due to opportunistic infections, neoplasm or related to drug toxicity. The present study was done to determine etiological factors, biochemical profile and outcome of patients of HIV with acute kidney injury (AKI) and chronic kidney disease (CKD) separately.

\section{MATERIALS AND METHODS}

In this descriptive study, HIV positive patients aged $>18$ years who were admitted or seen on OPD basis were screened for renal involvement Patients who were positive on screening were classified as AKI or CKD, underwent investigations for cause including kidney biopsy if indicated and followed up prospectively.

\section{RESULTS}

Total 116 patients were observed of which 82 had AKI, and 34 patients had CKD. Infectious diseases causing AKI was the most prevalent cause especially in patients not on ART followed by tenofovir (TDF) use causing AKI. 74/82 (90.24\%) had CD4 count of < 500 cells/mm3.

CKD group comprised of only CKD in 5 patients, TDF nephrotoxicity overlapping with CKD in 19 patients and AKI on CKD in 10 patients. 32/34 (94.11\%) had CD4 count of $<500$ cells $/ \mathrm{mm} 3$.

In total 84 (72.41\%) patients with renal failure comprising 54 (65.85\%) from AKI group and 30 (88.24\%) patients from CKD group were on TDF based ART regimen. In $5(6 \%)$ patients with TDF induced AKI had the partial recovery with high creatinine values at the time of discharge and 3 months follow up suggesting progression to CKD.

\section{CONCLUSION}

Prerenal AKI due to various opportunistic infections was a significant cause of AKI particularly in patients not on ART. Tenofovir was leading cause of renal failure causing AKI as well as contributing to nephrotoxicity in CKD cases. Thus, our study shows that drug-induced nephrotoxicity mainly due to TDF is a very important cause of morbidity in HIV. Our study showed a strong correlation between advanced immunosuppression and renal failure.

\section{KEYWORDS}

HIV associated Nephropathy, Tenofovir, Acute Kidney Injury, Chronic kidney disease.

HOW TO CITE THIS ARTICLE: Patil PV, Bansode JB, Kore MC, et al. Acute kidney injury and chronic kidney disease in HIV patients in the era of anti- retroviral therapy. J. Evolution Med. Dent. Sci. 2018;7(09):1082-1086, DOI: 10.14260/jemds/2018/246

\begin{abstract}
BACKGROUND
Implementation of ART has decreased the incidence of HIV Associated Nephropathy (HIVAN), but other causes of renal diseases persist and are increasing in prevalence. These may be Opportunistic Infectious(OIs) and other conditions related to HIV infection, medication adverse effects caused by some ART drugs, drugs used in the treatment of OIs and other co morbidities (e.g., diabetes mellitus, hypertension).[1] So, there is direct need to assess renal function in patients with HIV infection in India. Hence, we designed a study to evaluate clinical profile of HIV patients with renal failure. Moreover, we assessed etiological factors responsible for the renal
\end{abstract}

'Financial or Other Competing Interest': None.

Submission 19-01-2018, Peer Review 13-02-2018,

Acceptance 20-02-2018, Published 26-02-2018.

Corresponding Author:

Dr. Jyoti B. Bansode,

In Front of Kings Kingdom English School,

Hanuman Chowk, Jadhav Vadi Road,

Osmanabad-413501, Maharashtra.

E-mail: dr.jyotibansode.01@gmail.com

DOI: $10.14260 /$ jemds $/ 2018 / 246$

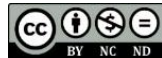

failure and renal recovery in such patients. Infections of gastrointestinal tract with protozoa such as cryptosporidia, microspora, isospora belli, bacteria such as salmonella, shigella, campylobacter can lead to diarrhoea and pre-renal AKI. Less common causes of AKI are direct infectious insults from HIV virus, immune restoration inflammatory syndrome(IRIS), rhabdomyolysis and obstruction, severe pancreatitis caused by various OIs (cytomegalovirus, cryptosporidiosis, mycobacterial) or drugs nucleoside reverse transcriptase inhibitors such as didanosine and stavudine and antitubercular drugs such as isoniazid. [2]

The dramatic increase in the use of TDF since its approval by the U. S. Food and Drug Administration in 2001 has prompted considerable interest in studying the incidence and severity of tenofovir nephrotoxicity. Multiple case reports of AKI, Fanconi syndrome, and nephrogenic diabetes insipidus associated with TDF use have been published. TDF nephrotoxicity is particularly relevant in the Indian context as National AIDS Control Organisation (NACO) launched TLE (TDF, Lamivudine, Efavirenz) regimen as first line regimen from year 2013 for all patients. Fanconi syndrome caused by TDF is characterized by generalized proximal tubular 
dysfunction resulting in one or more of the following: bicarbonaturia, glucosuria, phosphaturia, uricosuria, aminoaciduria, and tubular proteinuria. It is hypothesized that this toxicity may be a result of mitochondrial DNA depletion or direct tubular cytotoxicity. Episodes of AKI related to TDF may be irreversible and lead to End Stage Renal Disease (ESRD) if the drug is not promptly discontinued. Therefore, regular monitoring of kidney function and urine studies during TDF administration are recommended.

Agents known to cause acute interstitial nephritis, such as nonsteroidal Anti-inflammatory drugs (NSAIDs), trimethoprim-sulfamethoxazole, and rifampin are used frequently in HIV-infected patients. ${ }^{[3]}$ Amphotericin B used in treatment of cryptococcal infection in HIV-infected patients is known for its direct toxicity to renal tubular cells. Antitoxoplasmosis agent Sulfadiazine, Antiviral agent's acyclovir, indinavir, and foscarnet used in the treatment of OIs caused by HIV, are all reported to cause intrarenal crystal deposition can cause AKI. The protease inhibitor ritonavir also is associated with AKI from direct tubular injury.

HIV associated nephropathy (HIVAN) is synonymous with HIV associated chronic kidney disease (CKD). Prior to the presence of effective ART, HIVAN was so frequent with dramatic clinical features such as heavy proteinuria, and rapid progression to ESRD.[4] HIVAN is diagnosed by kidney biopsy. Focal and segmental glomerulosclerosis (FSGS) is the most common histological finding on renal biopsy. There may also be membranoproliferative glomerulonephritis, minimal change disease, membranous glomerulopathy, amyloidosis, acute immune-complex glomerulonephritis, cryoglobulinaemia, and IgA nephropathy on renal biopsy suggestive of HIVAN. Risk factors for renal disease in HIV patients include CD4 count $<200$ cells/microliter, HIV viremia particularly RNA levels $>400$ copies/ml,[5] African-American race, family history of kidney disease, use of nephrotoxins, comorbidities like diabetes, hypertension, hepatitis c co infection. If HIVAN is left untreated, it can progress to ESRD within weeks to months. Several retrospective analyses have determined the clinical benefit of various therapies, including the use of steroids, renin-angiotensin-aldosterone inhibitors, and HAART. The rationale behind the use of HAART is based on the decrease in the progression of HIV in the development of HIVAN, which is supported by the response to HAART in the case with HIVAN and relapse of disease with cessation of therapy.[6] The scientific reason behind the use of corticosteroids in individual with HIVAN is the significant tubulointerstitial inflammation present on histological examination of the renal tissue in these individual.[7] PreHAART, HIV infection was an absolute contraindication for the majority of organ transplantation. Currently, however, improvement in the overall survival rate of HIV infected individual has resulted in organ transplantation emerging as a potential therapeutic option for those with end-organ failure. HIV-1-infected individuals with ESRD are considered renal transplant candidates if their CD4 count is at least 200 cells/mm3 and HIV-1 is undetectable.[8]

Obstruction of the urinary system is a rare but noteworthy cause of renal failure in patients with HIV. Extrarenal compression of the ureters by retroperitoneal lymph nodes enlarged by infiltrating lymphoma or carcinoma cervix, an AIDS defining malignancy caused by human papilloma virus, an OI can lead to obstructive uropathy and renal failure. Sulfadiazine may crystallize in the kidney while Indinavir and Atazanavir may form renal calculi.

\section{Aims and Objectives}

To study clinical profile and etiological factors of HIV patients with renal failure (Acute and Chronic).

\section{MATERIALS AND METHODS}

The study began after obtaining permission from the Institutional Ethics Committee. All patients were explained the purpose and rational of the study as well as their role as participants in the study. This study was a descriptive study which was conducted for two years in Department of General Medicine of Tertiary Care Hospital.

\section{Patients with following Criteria were selected for this Study}

HIV positive patients aged $>18$ years with acute or chronic renal failure admitted or on OPD basis. Patients with following criteria were excluded from the study: HIV positive patients with CKD due to known causes like DM nephropathy, HTN nephropathy or connective tissue disease.

The definition of AKI in HIV-seropositive patients as per guidelines from the HIV Medicine Association of the Infectious Diseases Society of America (HIVMA-IDSA) 6 is creatinine level greater than $1.5 \mathrm{mg} / \mathrm{dL}$ or a 1.3 -fold increase above laboratory baseline that resolves within 3 months.[9] HIVMA-IDSA guidelines on HIV-related renal disease recommend use of the term chronic kidney disease as defined by the National Kidney Foundation, which refers to documented kidney damage present for 3 months or more and graded by calculating either the creatinine clearance using the modified Cockcroft-Gault equation or the glomerular filtration rate (GFR) by use of the modification of diet in the renal disease equation. A persistently reduced GFR $<60 \mathrm{~mL} / \mathrm{min} / 1.73 \mathrm{~m} 2$ signifies possible chronic kidney disease.[10]

Cockcroft-Gault equation: eGFR $=\{((140$-age $) \times$ weight $) /$ (72 x serum creatinine) $\} \times 0.85$ (if female)

The GFR is expressed in $\mathrm{mL} / \mathrm{min} / 1.73 \mathrm{~m} 2$

Total 116 Patients of HIV with renal failure were studied. Detailed evaluation including history, WHO clinical staging of the study population at the time of visit, CD4 count, general examination and clinical examination, investigations were performed. ART data were obtained from patients admitted in medicine wards, from ART centre, from ART registration book, from SACEP records (State AIDS Clinical Expert Panel) meetings held at Centre of Excellence J J Hospitals, Mumbai with NACO permission and from previous case paper records if any available. Renal biopsy was done with informed consent where indicated and results were analysed. The information was recorded after obtaining informed consent from patient and kept confidential.

\section{RESULTS}

Total 116 patients were observed. $82(70.68 \%)$ patients had AKI and 34 (29.31\%) patients had developed CKD. In this study, we found mean age of the HIV patients with AKI was $35.33+9.76$ years and with CKD was $41.1 \pm 11.1$ years, AKI cases were observed most commonly in 4 th and 5 th decades of age and CKD in 5th and 6th decades of age. There was male preponderance with male: female ratio of $2.5: 1$ 
The etiological spectrum for AKI being tenofovir in 33 (40.24\%) patients, TDF with infectious causes in 14 (17\%) and only infectious causes in 35 (42.68\%) patients.

35 patients $(42.68 \%)$ who had AKI only due to infectious causes as well as $14(17 \%)$ patients with TDF with infectious causes; had complete recovery (with normal creatinine) at the time of discharge. In the group of patients with only TDF induced AKI of 33 (40\%) patients, 28 (34.14\%) patients showed complete recovery at the time of discharge, but $5(6.09 \%)$ patients had partial recovery with high creatinine values at the time of discharge and at 3 months follow up suggesting progression to CKD. Infectious diseases causing AKI was the most prevalent cause (Specially in patients not on ART) followed by TDF use causing AKI (in patients on ART).

CKD group comprised of only CKD in $5(14.70 \%)$ patients, TDF nephrotoxicity overlapping with CKD in 19 (55.88\%) patients and AKI on CKD in 10 (41.66\%) patients. Two $(1.76 \%)$ patients with HIVAN were diagnosed in our study, one presented with AKI and the other with AKI on CKD and both had proteinuria.

In total $84(72.41 \%)$ patients with renal failure comprising 54 (65.85\%) from AKI group and 30 (88.24\%) from CKD group, TDF was being used as an ART regimen with mean duration of tenofovir use was $15.11+12.5$ months for the AKI group and 16.31+ 14.77 months for the CKD group.

In AKI group 74/82 (90.24\%) had a CD4 count of $<500$, and In CKD group, 32/34 (94.11\%) had a CD4 count of $<500$ indicating an association of immunosuppression with renal failure. In AKI group $71.95 \%$ patients were with WHO stage II, III, IV (symptomatic HIV disease) and $29.27 \%$ were asymptomatic and in CKD group, $61.77 \%$ were with WHO stage II, III and IV. 2 (2.38\%) patients had Fanconi's syndrome in our study characterized by renal glycosuria and hypophosphatemia which is suggestive of tenofovir induced tubulopathy.

In our study, renal biopsy was performed in 6 patients of renal failure, 2 from AKI group and 4 from CKD group. Results showed tubulointerstitial disease in 3, HIVAN or collapsing nephropathy in 2 and interstitial nephritis with glomerulosclerosis in 1 patient.

\begin{tabular}{|c|c|c|c|c|}
\hline ART & AKI & AKI (\%) & CKD & CKD (\%) \\
\hline No ART & 10 & 12.20 & 00 & 0.00 \\
\hline $\begin{array}{c}\text { ZLN/ZLE / ZL Atv r/ } \\
\text { ZL Lpv r }\end{array}$ & 12 & 14.63 & 01 & 2.94 \\
\hline SLN / SLE / SL Lpv / r & 01 & 1.22 & 03 & 8.82 \\
\hline $\begin{array}{c}\text { TLN / TLE / TL Atv r/ } \\
\text { Tl Lpv r /Abc LE }\end{array}$ & 54 & 65.85 & 30 & 88.24 \\
\hline Defaulter & 05 & 6.10 & 00 & 0.00 \\
\hline Total & $\mathbf{8 2}$ & $\mathbf{1 0 0 . 0 0}$ & $\mathbf{3 4}$ & $\mathbf{1 0 0 . 0 0}$ \\
\hline \multicolumn{5}{|c}{ Table 1. ART } \\
\hline
\end{tabular}

\begin{tabular}{|c|c|c|c|c|}
\hline $\begin{array}{c}\text { CD4 count } \\
\text { (cells/mm3) }\end{array}$ & AKI (n) & AKI (\%) & CKD (n) & CKD (\%) \\
\hline$<200$ & 32 & 39.02 & 18 & 52.94 \\
\hline $200-500$ & 42 & 51.22 & 14 & 41.18 \\
\hline$>500$ & 08 & 9.76 & 02 & 5.88 \\
\hline Total & $\mathbf{8 2}$ & $\mathbf{1 0 0 . 0 0}$ & $\mathbf{3 4}$ & $\mathbf{1 0 0 . 0 0}$ \\
\hline \multicolumn{5}{|c|}{ Table 2. CD4 count } \\
\hline
\end{tabular}

\begin{tabular}{|c|c|c|}
\hline Diagnosis & $\mathbf{n}$ & $\%$ \\
\hline Tenofovir induced AKI & 34 & 41.46 \\
\hline \multicolumn{3}{|c|}{$\begin{array}{l}\text { Tenofovir Induced AKI in Addition to } \\
\end{array}$} \\
\hline Acute Gastroenteritis & 9 & 12.20 \\
\hline Urinary tract infection & 02 & 2.44 \\
\hline Acute pancreatitis & 01 & 1.22 \\
\hline Malaria & 01 & 1.22 \\
\hline Acute Febrile Illness & 01 & 1.22 \\
\hline \multicolumn{3}{|c|}{ AKI due to } \\
\hline Acute Gastroenteritis & 21 & 25.61 \\
\hline Urinary tract infection & 01 & 1.22 \\
\hline Acute pancreatitis & 03 & 3.66 \\
\hline Pneumonia & 02 & 2.44 \\
\hline Malaria & 06 & 7.32 \\
\hline Acute Febrile Illness & 01 & 1.22 \\
\hline Total & 82 & 100.00 \\
\hline \multicolumn{3}{|c|}{ Table 3. Final diagnosis in AKI patients } \\
\hline
\end{tabular}

\begin{tabular}{|c|c|c|}
\hline Diagnosis & $\mathbf{n}$ & $\%$ \\
\hline Cause undefined & 01 & 2.94 \\
\hline Small sized kidneys & 04 & 11.76 \\
\hline Tenofovir causing CKD & 19 & \\
\hline \multicolumn{3}{|c|}{ CKD with AKI due to } \\
\hline Urinary tract infection & 06 & 17.65 \\
\hline Acute Gastroenteritis & 02 & 5.88 \\
\hline Acute Pancreatitis & 01 & 2.94 \\
\hline Abdominal Tuberculosis & 01 & 2.94 \\
\hline Total & 34 & 100.00 \\
\hline \multicolumn{3}{|c|}{ Table 4. Final diagnosis in CKD patients } \\
\hline
\end{tabular}

\section{DISCUSSION}

HIV infection is the modern pandemic. In our study of 116 patients presenting with renal failure, 82 had AKI, and 34 had CKD. There was male preponderance with male: female ratio of 2.5:1. Prakash J et al, 2015 reported that there were 76.08\% HIV infected males and $23.18 \%$ HIV infected females diagnosed with AKI. These reports were similar to our study.[11]

From this study 54 (65.85\%) patients from AKI group and $30(88.24 \%)$ patients from CKD group who had AKI were on ART regimen in which TDF was present -TDF with NNRTI combination i.e. TL+E/N or TDF with Protease Inhibitor i.e.TL Atv/r or TL Lpv/r. Thus, the mechanism of AKI in 84/116 (73.68\%) had TDF induced AKI. Thus, our study indicates that AKI due to TDF induced tubule interstitial disease is an important mechanism and the incidence of AKI is therefore much less in TDF sparing regimen.[12] 5(6.09\%) patients with AKI who were on TDF based regimen had a partial recovery with high creatinine values at the time of discharge and at 3 months follow up suggesting progression to CKD.

Manosuthi W showed the potentially irreversible adverse effect of tenofovir.[13]

In AKI group, CD4 count of $<200$ was in 32 (39.02\%), $200-500$ in $42(51.22 \%)$ and $>500$ in $8(9.76 \%)$ patients. Prakash J et al shows that majority of cases were in advance stages of HIV infection with the mean CD4 count of $201.38 \pm$ $151.22 \mathrm{cell} / \mu \mathrm{l}$ and 85 (61.59\%) patients had CD4 count $<200$ cell $/ \mu$ l. 11 which correlates with our study. In CKD group, CD4 count of <200 was in 18 (52.94\%) patients, 200-500 in $14(41.18 \%)$ patients and $>500$ in $2(5.88 \%)$ patients.

Ayokunle DS et al, study shows that in CKD patients with HIV HAD lower CD4 T-cell count and a greater proportion (57.4\%) had CD4 T-cell count $<200$ cells/ $\mu$ l for CKD.[14] 
Advanced immunosuppression is itself is a risk for renal failure as HIV virus can cause mitochondrial toxicity.

In our study, 34 patients were diagnosed to have CKD on the basis of documented previous elevated serum creatinine value atleast 3 months prior, at the time of inclusion in our study.

The final diagnosis in CKD patients was categorized into three groups.

First group: patients with only chronic kidney disease 5 $(14.70 \%)$ in whom bilateral small sized kidneys were seen on ultrasonography of abdomen +KUB.

The second group which comprised of 19 (55.88\%) patients with CKD in whom tenofovir was administered in adjusted doses due to high creatinine values at baseline.

The Third group comprised of AKI due to various causes with underlying CKD with or without overlapping TDF use. It includes $3(8.82 \%)$ patients with Ca cervix with obstructive uropathy, 1 (2.94\%) patient with polycystic kidney disease, 1 (2.94\%) patient with HIVAN and 5(14.70\%) patients with TDF induced CKD.

$30(88.24 \%)$ patients from CKD group were on ART regimen in which TDF contributed to significant nephrotoxicity in these patients.

In patients with tenofovir with CKD, ART regimen was changed in $14 / 19(73.68 \%)$ patients to stavudine or abacavir based regimens. In many of these patients, tenofovir was already being administered in adjusted doses by some practitioners for variable periods before registration at our ART center. In many of such patients, there was a steady increase in the creatinine values from the baseline on the tenofovir regimen and hence their regimens were eventually changed to stavudine/abacavir after admission at our institution or registration at our ART center. 2 (10.52\%) patients who were HBsAg positive continued on the tenofovir based regimen with dose adjusted according to creatinine clearance as both had the chronic liver disease due to hepatitis B and hence tenofovir was very essential for treatment of liver disease.

In patients with AKI on CKD group 9/10 in (90\%) of cases tenofovir regimen was changed to abacavir / stavudine based regimen. In $1(10 \%)$ patient tenofovir was continued in adjusted dose as the patient was HBsAg positive.

Stavudine was phased out with new guidelines of TLE regimen as $1^{\text {st }}$ line ART from September 2012 at all ART centers across India.

In the initial period of these changes, the exact policy of tenofovir usage in situations where baseline creatinine is high was unclear, and there was no awareness of toxicity profile in ART medical officers. Hence, in some cases, tenofovir was started/continued in CKD patients after adjustment of doses according to creatinine clearance. Hence, in this scenario tenofovir was continued in adjusted doses rather than changing regimen. Such patients developed irreversible nephrotoxicity due to TDF.

Renal biopsy was performed in 2 patients of AKI and 4 patients of CKD. The biopsy results showed tubulointerstitial disease in 3, collapsing glomerulopathy in 2 and interstitial nephritis with glomerulosclerosis in 1 patient.

\section{In AKI Group}

\section{Case 1}

Patient had AKI due to UTI, and acute pancreatitis with Hepatitis B. He had subnephrotic range proteinuria which persisted after resolution of AKI. Hence renal biopsy was performed which showed severe tubulointerstitial disease.

\section{Case 2}

Patient had AKI due to AGE. However, the biopsy was done for nephrotic range proteinuria. Biopsy showed minimal change disease. The pathological changes of FSGS are most prominent in glomeruli located at the corticomedullary junction, so if the renal biopsy specimen is from superficial tissue, the lesion can be missed which sometimes leads to a misdiagnosis of minimal change disease.

\section{In CKD Group-}

Case 1

Patient had subnephrotic range proteinuria with CKD with normal sized kidneys. Hence renal biopsy was done which showed classical collapsing glomerulopathy suggestive of HIVAN.

\section{Case 2 and 3}

Renal biopsy was done due to subnephrotic range proteinuria. These patients were on the tenofovir based regimen, the biopsy revealed interstitial nephritis with glomerulosclerosis which could be due to TDF.

Thus 2 out of 3 cases with CKD in HIV had tenofovir as the cause.

\section{CONCLUSION}

In our series, tenofovir was leading cause of renal failure causing AKI as well as contributing to nephrotoxicity in CKD cases. Prerenal AKI due to various infections was also a significant cause of AKI particularly in patients not on ART. HIVAN was also found in only small minority of cases. Thus, our study shows that drug induced nephrotoxicity mainly due to tenofovir is a very important cause of morbidity in HIV patients and our study highlights the impact of tenofovir specially in the recent era where stavudine was phased out and replaced by tenofovir as the first line regimen. Thus, our study underlines the need of careful monitoring of patients on tenofovir based ART regimen and regular monitoring with creatinine levels, urine examination for proteinuria and glycosuria. Detection of these abnormalities will prompt immediate change to other regimens preventing significant morbidity. Even in cases of CKD choosing tenofovir sparing regimen in favour of continuation of tenofovir with creatinine clearance adjusted doses may prevent further nephrotoxicity.

\section{REFERENCES}

[1] De Silva TI, Post FA, Griffin MD, et al. HIV-1 Infection and the kidney: an Evolving Challenge in HIV Medicine. Mayo Clinic Proceedings 2007;82(9):110316.

[2] Kalim S, Szczech L, Wyatt CM. Acute kidney injury in hiv-infected patients. Seminars in Nephrology 2008;28(6):556-62.

[3] HIV InSite. Choice Reviews Online. 2003;40(12):40Sup-0297-40Sup-0297.

[4] Winston J, Deray G, Hawkins T, et al. Kidney disease in patients with hiv infection and AIDS. Clinical Infectious Diseases 2008;47(11):1449-57. 
[5] Estrella M, Fine DM, Gallant JE, et al. HIV type 1 RNA level as a clinical indicator of renal pathology in HIV-infected patients. Clinical Infectious Diseases 2006;43(3):377-80.

[6] Julia SJ, Mohamed AG, Derek FM. Relapse of HIVassociated nephropathy after discontinuing highly active antiretroviral therapy. AIDS 2007;21(2):263-4.

[7] Briggs WA, Tanawattanacharoen S, Choi MJ, et al. Clinicopathologic correlates of prednisone treatment of human immunodeficiency virus-associated nephropathy. American Journal of Kidney Diseases 1996;28(4):618-21.

[8] Atta MG, Lucas GM, Fine DM. HIV-associated nephropathy: epidemiology, pathogenesis, diagnosis and management. Expert Review of Anti-Infective Therapy 2008;6(3):365-71.

[9] Gupta SK, Eustace JA, Winston JA, et al. Guidelines for the management of chronic kidney disease in HIVinfected patients: recommendations of the HIV Medicine Association of the Infectious Diseases Society of America. Clinical Infectious Diseases 2005;40(11):1559-85.
[10] Levey AS, Coresh J, Balk E, et al. National Kidney Foundation practice guidelines for chronic kidney disease: evaluation, classification, and stratification. Annals of Internal Medicine 2003;139(2):137-47.

[11] Prakash J, Gupta T, Prakash S, et al. Acute kidney injury in patients with human immunodeficiency virus infection. Indian Journal of Nephrology 2015;25(2):86-90.

[12] Zaidan M, Lescure FX, Brocheriou I, et al. Tubulointerstitial nephropathies in HIV-infected patients over the past 15 years: a clinico-pathological study. Clinical Journal of the American Society of Nephrology 2013;8(6):930-8.

[13] Manosuthi W, Mankatitham W, Lueangniyomkul A, et al. Renal impairment after switching from stavudine/lamivudine to tenofovir/lamivudine in NNRTI-based antiretroviral regimens. AIDS Research and Therapy 2010;7(1):37.

[14] Ayokunle DS, Olusegun OT, Ademola A, et al. Prevalence of chronic kidney disease in newly diagnosed patients with Human immunodeficiency virus in Ilorin, Nigeria. J Brasileiro de Nefrologia 2015;37(2):177-84. 